\title{
Modelo de nivel de madurez para los procesos de emprendimiento en las pymes colombianas
}

\author{
Maturity Level Model for Entrepreneurship \\ Processes in Colombian smes
}

\author{
Laura Camila Nungo-Pinzón ${ }^{1} \bowtie$, Brayan Torres-González², \\ José Ignacio Palacios-Osma ${ }^{3}$
}

\author{
1 Universidad Distrital Francisco José de Caldas, Bogotá, Colombia. \\ ${ }^{2}$ Universidad Distrital Francisco José de Caldas, Bogotá, Colombia \\ ${ }^{3}$ Universidad Distrital Francisco José de Caldas, Bogotá, Colombia
}

Gicoecol (Grupo de investigación en Comercio Electrónico Colombiano), Facultad de Ingeniería Industrial, Universidad Distrital Francisco José de Caldas, Carrera 7 n. ${ }^{\circ}$ 40B-53, Bogotá, Colombia.

Correo electrónico: lacnungop@correo.udistrital.edu.co.

Recibido: febrero 5 del $2018 \quad$ Aprobado: junio 15 del $2018 \quad$ Disponible en línea: septiembre 1 del 2018

Cómo citar este artículo: L. C. Ñungo-Pinzón, B. Torres-González, J. I. Palacio-0sma, “Modelo de madurez para los procesos de emprendimiento en las pymes colombianas", Revista Ingeniería Solidaria, vol. 14, no. 26, 2018. doi: https://doi. org/10.16925/in.v14i26.2456

\section{Resumen}

Introducción: esta investigación presenta una propuesta de modelo de madurez aplicado a los procesos de emprendimiento en las pymes colombianas, realizada durante el 2017 en la Universidad Distrital Francisco José de Caldas. Su objetivo es hacer un diagnóstico de la acción emprendedora en este tipo de organizaciones a través de la evaluación de cinco factores: perfil del emprendedor, aspectos económicos y financieros, condiciones del mercado, creación y difusión del conocimiento y soluciones tic, y relacionarlos con los diferentes niveles de madurez planteados por la metodología cмmı (Capability Maturity Model Integration).

Metodología: a) se hace una revisión de la literatura referente a los temas de emprendimiento y modelo de madurez, b) se identifican los principales factores para los procesos de emprendimiento, c) se diseña el modelo de madurez, d) se valida el modelo por medio de la metodología Delphi y juicio de expertos, e) se lleva acabo el análisis estadístico de los resultados de las pruebas realizadas, y f) se plantean las conclusiones y acciones de mejora para incrementar el nivel de madurez del factor "condiciones del mercado", desarrollado a lo largo del escrito.

Resultados: el modelo de madurez validado muestra un porcentaje de aceptación de los expertos del $83,75 \%$, sugiriendo algunas mejoras en los niveles propuestos.

Conclusión: este modelo muestra la situación de los procesos de emprendimiento para empresas ya constituidas, y mediante su implementación por un experto pretende generar acciones de mejora y lograr que el emprendimiento sea una herramienta para el crecimiento económico de la organización.

Limitaciones: el modelo propuesto no ha sido probado en una pyme y se encuentra hasta este momento en su construcción conceptual.

Originalidad: no se ha establecido un modelo similar que evalúe los procesos de emprendimiento en las empresas a través de la metodología cMmI.

Palabras clave: diagnóstico del emprendimiento empresarial, emprendimiento, factores de emprendimiento, modelo de madurez. 


\title{
Maturity Level Model for Entrepreneurship Processes in Colombian smes
}

\begin{abstract}
Introduction: This research, conducted in 2017 at the Universidad Distrital Francisco José de Caldas, presents a proposed maturity model applied to entrepreneurial processes in Colombian smes. Its aim is to diagnose the entrepreneurial action in these organizations by assessing five factors: entrepreneur profile, economic and financial aspects, market conditions, knowledge creation and dissemination, and Ict solutions, and relate them to various maturity levels proposed by the сммі (Capability Maturity Model Integration) method.
\end{abstract}

Methods: a) Literature referring to entrepreneurship and maturity models is reviewed; b) the main factors for entrepreneurship processes are identified; c) the maturity model is designed; d) the model is validated by means of the Delphi method and expert judgment; e) the statistical analysis of tests results is carried out; and f) conclusions and improvement actions are proposed to increase the maturity level of the "market conditions" factor, which is elaborated throughout the document.

Results: The validated maturity model shows an experts' acceptance percentage of $83.75 \%$, suggesting some improvements to the proposed levels.

Conclusion: This model shows the situation of entrepreneurship processes for already incorporated companies and, through its implementation by an expert, intends to create improvement actions and make entrepreneurship a tool for the organization's economic growth.

Limitations: The proposed model has not been tested in an sme and is currently in its conceptual construction. Originality: A similar model has not been established to assess entrepreneurial processes in companies using the cmm method.

Keywords: business entrepreneurship diagnosis, entrepreneurship, entrepreneurship factors, maturity model.

\section{Modelo de nível de maturidade para processos de empreendedorismo em PMEs colombianas}

\section{Resumo}

Introdução: esta pesquisa apresenta uma proposta de modelo de maturidade aplicado aos processos empreendedores das PMEs colombianas, realizado durante 2017 na Universidad Distrital Francisco José de Caldas. 0 objetivo é fazer um diagnóstico da ação empreendedora nesse tipo de organização através da avaliação de cinco fatores: perfil empreendedor, aspectos econômicos e financeiros, condições de mercado, criação e disseminação de conhecimento e soluções de tic; e relacioná-los a diferentes níveis de maturidade propostos pela metodologia cMMI (Capability Maturity Model Integration).

Metodologia: a) faz-se uma revisão da literatura referente ao modelo de empreendedorismo e maturidade, b) identificam-se os principais fatores para os processos de empreendedorismo, c) desenha-se o modelo de maturidade, d) valida-se o modelo por meio da metodologia Delphi e julgamento de especialistas, e) realiza-se a análise estatística dos resultados dos testes aplicados, e f) retiram-se as conclusões e ações de melhoria para aumentar o nível de maturidade do fator "condições de mercado", desenvolvido ao longo do texto.

Resultados: o modelo de maturidade validado mostra uma porcentagem de aceitação dos especialistas de $83,75 \%, 0$ que sugere algumas melhorias nos níveis propostos.

Conclusão: este modelo mostra a situação dos processos de empreendedorismo para empresas já estabelecidas. Através de sua implementação por um especialista, o modelo busca gerar ações de melhoria e tornar o empreendedorismo uma ferramenta para o crescimento econômico da organização.

Limitações: o modelo proposto não foi testado em uma PME e atualmente está em sua construção conceitual. Originalidade: nenhum modelo semelhante foi estabelecido para avaliar os processos de empreendedorismo nas empresas por meio da metodologia cмmı.

Palavras-chave: diagnóstico de empreendedorismo empresarial, empreendedorismo, fatores de empreendedorismo, modelo de maturidade. 


\section{Introducción}

En Colombia, según Ángel y Pulido [1], las pymes juegan un papel importante en la actividad económica, lo cual impulsa el crecimiento y el desarrollo de ideas innovadoras que facilitan los procesos de emprendimiento. La perspectiva actual de las pymes colombianas establece ciertos desafíos que deben ser tomados en cuenta para iniciar nuevas estrategias que vayan encaminadas a la mejora continua y al crecimiento sostenible de estas empresas. Algunos de los retos que tienen actualmente las pymes son: el fortalecimiento de su recurso humano o cultura organizacional (Salas-Arbeláez et al. [2]), la transferencia del conocimiento (Lis-Gutiérrez et al. [3]), las mejoras en calidad y productividad, la creación de redes de negocios (clusters), el desarrollo de actividades de $\mathrm{I}+\mathrm{D}+\mathrm{i}$ que contribuyan a la generación de innovación, la expansión en el mercado nacional e internacional y la optimización de sus procesos (Varela-Villegas [4]).

De acuerdo con García-Pérez de Lema et al. [5], las ideas nuevas e innovadoras se han convertido en recursos estratégicos para las pymes, que han podido buscar y desarrollar conocimiento a través de procesos de emprendimiento con el fin de mejorar su competitividad e impactar positivamente los niveles de crecimiento económico [5]. Actualmente, como mencionan Escandón-Barbosa y Hurtado-Ayala [6], la actividad emprendedora en Colombia está caracterizada por la creación de nuevas empresas que buscan la identificación y el aprovechamiento de oportunidades existentes en el mercado que permitan impulsar la actividad empresarial en el país. No obstante, el proceso emprendedor también es desarrollado en empresas ya consolidadas, con el fin de afianzar su posición en el mercado generando crecimiento económico por medio de la innovación en procesos y productos, según Pereira [7].

Diferentes autores, como Galindo-Martín et al. [8] y Kantis et al. [9], han demostrado la relación directa que existe entre el emprendimiento y el crecimiento económico y empresarial en los países [8], razón por la cual es importante identificar las características, los factores y los objetivos que debe tener cualquier proceso emprendedor [9]. La medición de estos procesos se puede realizar por medio de un modelo de madurez que cumpla los propósitos empresariales y que contenga los elementos esenciales para el mejoramiento y la eficiencia en una o más áreas [10].

Estos modelos describen un camino de mejora progresiva que permite pasar de procesos inmaduros a procesos maduros de mejor calidad y más eficaces, categorizando cada uno de estos procesos en cinco niveles básicos según su nivel de madurez, como lo plantea Backlund et al. [11].

Esta investigación se desarrolló teniendo en cuenta principalmente la actividad empresarial de las pymes colombianas, su importancia e impacto en la economía, y el crecimiento del espíritu emprendedor evidenciado en este tipo de empresas. Por lo tanto, este artículo presenta una propuesta de modelo de madurez para los procesos de emprendimiento en las pymes colombianas, desarrollado mediante la construcción de un marco teórico, la definición de factores y niveles para los procesos de emprendimiento, y la validación de un modelo de madurez.

Este modelo permitirá identificar las condiciones de las pymes para enfrentar los retos actuales de la economía digital y la globalización, así como definir planes de mejoramiento orientados al posicionamiento organizacional.

\section{Antecedentes de la investigación}

\subsection{Antecedentes de los modelos de madurez}

Puello [12], en su investigación del 2013 "Modelo de verificación y validación basado en CMмI", hace una descripción cronológica de los modelos de madurez y muestra que sus orígenes se remontan a 1984 cuando el Congreso de Estados Unidos aprobó la creación de un organismo de investigación para el desarrollo de modelos de mejora llamado Instituto de Ingeniería del Software (SEI, por sus siglas en inglés).

En el 2012, Kishore et al. [13] realizaron una conceptualizacion de los CMMI [13], mencionando que hacia 1991 el SEI, con ayuda del Carnegie Mellonde y la Universidad de Pittsburgh, publicó el primer modelo de madurez de capacidad aplicado a software (sw-CMM). Sutherland et al. [14], en el artículo del 2008 "Scrum and сммі Level 5: The Magic Potion for Code Warriors", describen este primer modelo como un método para mejorar la organización a través de comparaciones entre 
organizaciones disciplinadas o maduras e indisciplinadas o inmaduras.

A partir de este modelo, según lo planteado por De Vasconclelos et al. en el 2012 [15], se empezaron a crear diversos modelos de madurez, entre ellos tres que surgieron a mediados de los noventa:

- Desarrollo de productos y servicios (CMMI-DEV)

- Establecimiento y gestión de servicios (CMMIsvc)

- Adquisición de productos y servicios (CMMIACQ)

Como lo mencionan Peña-Forero y Aguirre Mayorga [16] en su investigacion del 2013 "Propuesta de diseño de un modelo de gestión basado en la integración de herramientas de tecnología de informacion (IT) a partir del análisis del estado de madurez de procesos (CMMI) para micro, pequeñas y medianas empresas (mipymes) en Bogota", la integración de estos procesos hacia finales de los noventa y hasta el año 2002 fue llamado CMMI. Este modelo de estructura compatible para todas las disciplinas evitó seguir implementando múltiples modelos por cada área de estudio. De acuerdo con Mejía et al. en el 2017 [17], el Сммі es el modelo más extendido, reconocido e implementado a nivel internacional dentro de las organizaciones de desarrollo de software, con el objetivo de elevar su nivel de madurez en la cultura de procesos y así obtener mayor calidad en los productos resultantes.

Según el estudio realizado por los investigadores Arboleda, Paz y Casallas en el 2013 [18], el CMMI ayuda a resolver problemas típicos de las organizaciones, tales como productos y servicios que no satisfacen los requerimientos del cliente, demoras y baja rentabilidad en los proyectos, altos costos operacionales, baja productividad, bajos niveles de innovación y desmotivación del personal, mediante la evaluación de factores concretos identificados en la organización versus niveles de madurez definidos, como lo mencionan Zapata-Jaramillo et al. [19] en su publicación del 2015 "Representation of CMMI-DEv Practices in the Semat Kernel," [19]

Garzás et al. [20], en su investigación del 2009 "Una aplicación de la norma ISO/IEC 15504 para la evaluación por niveles de madurez de pymes y pequeños equipos de desarrollo", exponen algunos de los modelos смм desarrollados en la ingeniería de software que más se destacan a nivel mundial: el ESSI (European Software and System
Initiative) para el caso de la Unión Europea; los modelos MoProSoft y EvalProSoft para México principalmente; el modelo ITMARK del Instituto Europeo del Software (ESI, por sus siglas en inglés) y el proyecto COMPETISOFT para Iberoamérica.

Aunque los modelos CMMI se han desarrollado principalmente para la ingeniería de software, su adaptabilidad y facilidad de implementación han permitido que puedan ser utilizados en otras disciplinas, con el fin de proporcionar una ayuda para la medición, evaluación y mejora de diversos procesos organizacionales. A continuación, se muestran algunos ejemplos de modelos CMMI desarrollados en diferentes áreas como: educación (modelo de acreditación institucional), tecnologías de la información (alineación estratégica de Laura Luftman) y simulación (modelo de madurez para evaluar la capacidad de adaptación de las organizaciones al contexto del Sistema de Fabricación Inteligente -IMS, por sus siglas en inglés-).

- Modelo de acreditación institucional basado еn сммі: este modelo de apoyo al proceso de acreditación, elaborado por Petrie et al. [21] y expuesto en una de sus publicaciones en el 2009, busca mejorar la capacidad de estos procesos en las instituciones de educación superior que pertenecen al LACCEI (Latin American and Caribbean Consortium of Engineering Institutions), facilitando la certificación de sus facultades y programas de Ingeniería. Propone cinco niveles de mejora continua y el cumplimiento de dos factores principales (capacidad y procesos) para que las instituciones puedan cumplir con los más altos requisitos de calidad (Figura 1).

- Modelo de madurez de alineación estratégica de Laura Luftman enfocado a la articulación de TI (tecnologías de la información) en las organizaciones: el modelo presentado por Laura Luftman [19] en el 2001 permite realizar una evaluación de la alineación estratégica entre las TI y las estrategias organizacionales por medio de cinco niveles planteados que van desde una etapa inicial (nivel 1; ausencia total del proceso), hasta una etapa de optimización (nivel 5; mejora continua de procesos). Adicionalmente, da a conocer la aplicación detallada de cada uno de los niveles mencionados, con el fin de determinar el nivel de madurez en el que se encuentra la 
organización o sector frente a la apropiación y el uso de las TI, y de ser necesario, que esta conozca los requerimientos que debe cumplir para adaptarse a un nivel específico (Tabla 1).

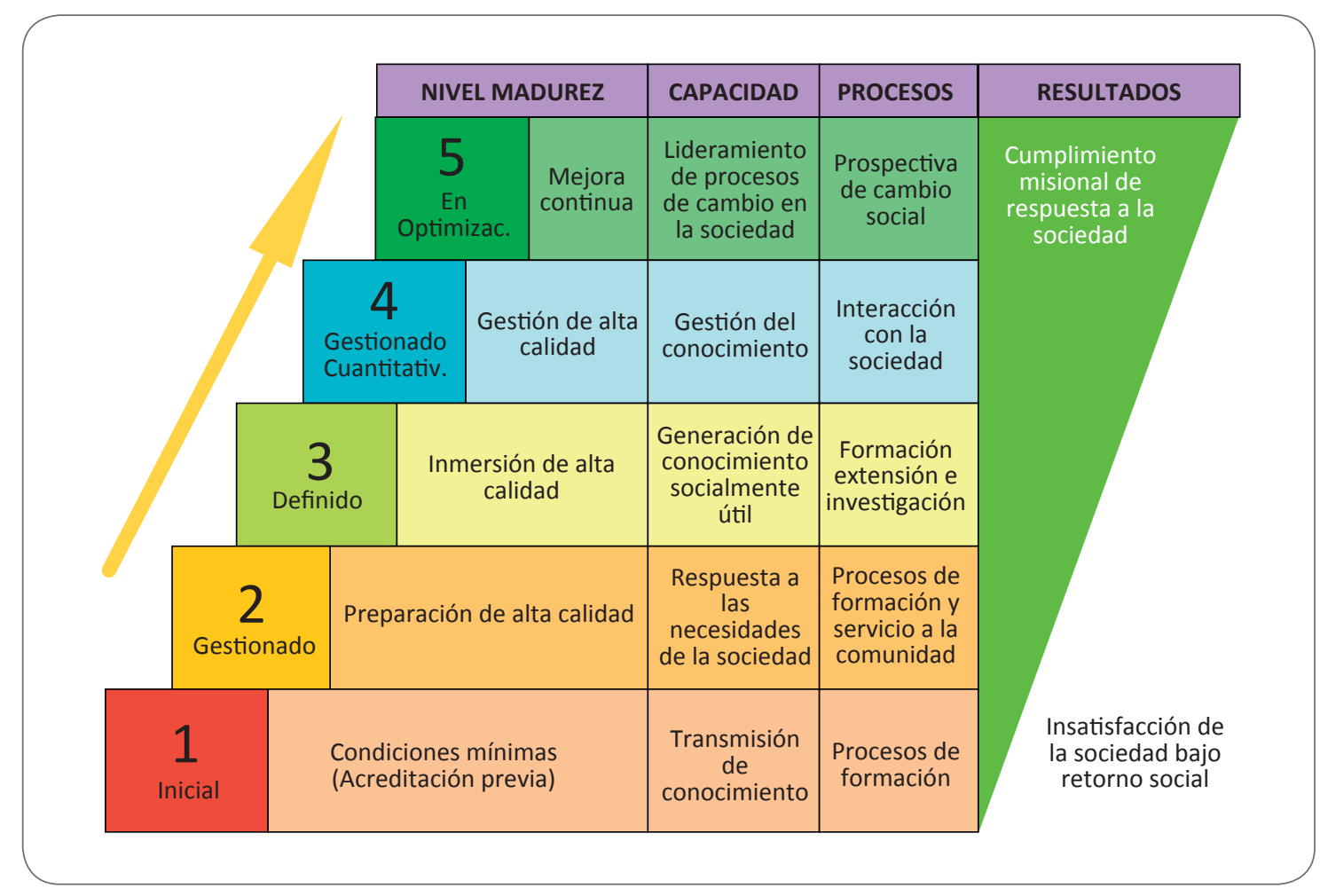

Figura 1. Modelo de acreditación institucional basado en CMмI Fuente: Petrie et al. [21]

Tabla 1. Modelo de madurez de alineación estratégica de Laura Luftman enfocado a la articulación de Ti en las organizaciones

\begin{tabular}{|c|c|c|}
\hline $\begin{array}{l}\text { Nivel de madurez } \\
\text { de representación } \\
\text { organizado (etapas) }\end{array}$ & Procesos & $\begin{array}{l}\text { Aplicación al alineamiento estratégico } \\
\text { de las TI por Laura Luftman }\end{array}$ \\
\hline Inicial (Nivel 1) & $\begin{array}{l}\text { Ausencia total de procesos definidos } \\
\text { (pobre control y sin previsión). }\end{array}$ & $\begin{array}{l}\text { Se encuentran las organizaciones con bajo } \\
\text { grado de madurez y poca alineación entre } \\
\text { sus estrategias y las TI. }\end{array}$ \\
\hline Gestionado (Nivel 2) & $\begin{array}{l}\text { Procesos de administración estable- } \\
\text { cidos para lograr el seguimiento de } \\
\text { costos, tareas y funcionalidad (hay } \\
\text { previsión algunas veces). }\end{array}$ & $\begin{array}{l}\text { Se encuentran las organizaciones que } \\
\text { tienen un compromiso inicial de alineación } \\
\text { entre sus estrategias y las TI. Cabe resaltar } \\
\text { que los procesos son de difícil alineación } \\
\text { debido a que el manejo de las Ti no los } \\
\text { realiza la organización. }\end{array}$ \\
\hline Definido (Nivel 3) & $\begin{array}{l}\text { Además de procesos de administra- } \\
\text { ción, se incluye la estandarización, } \\
\text { documentación y normalización de } \\
\text { estos (procesos organizados y con } \\
\text { previsión). }\end{array}$ & $\begin{array}{l}\text { Se encuentran las organizaciones que } \\
\text { tienen una alineación estratégica enfocada } \\
\text { en las Ti (con atributos concernientes a } \\
\text { comunicaciones, gobernabilidad y procesos } \\
\text { para la toma de decisiones). }\end{array}$ \\
\hline
\end{tabular}




\begin{tabular}{cll}
\hline $\begin{array}{c}\text { Nivel de madurez } \\
\text { de representación } \\
\text { organizado (etapas) }\end{array}$ & \multicolumn{1}{c}{ Procesos } & $\begin{array}{c}\text { Aplicación al alineamiento estratégico } \\
\text { de las TI por Laura Luftman }\end{array}$ \\
\hline Administrado (Nivel 4) & $\begin{array}{l}\text { Se llevan a cabo los proyectos en } \\
\text { forma controlada con métricas que } \\
\text { permiten mediciones confiables de } \\
\text { los procesos y productos. }\end{array}$ & $\begin{array}{l}\text { En este nivel, las organizaciones tienen } \\
\text { una gran gestión estratégica de las TI; la } \\
\text { dirección tiene propósitos de fortalecer el } \\
\text { concepto de Ti como valor central de la } \\
\text { organización y la gestión de TIC suele ser } \\
\text { una ventaja competitiva. }\end{array}$ \\
Optimizado (Nivel 5) & $\begin{array}{l}\text { Incluye la mejora continua de pro- } \\
\text { cesos a partir de la comparación y el } \\
\text { análisis de mediciones sucesivas de } \\
\text { los proyectos. }\end{array}$ & $\begin{array}{l}\text { En este nivel, las organizaciones han alcan- } \\
\text { zado un alto grado de alineación entre TI } \\
\text { ylas estrategias empresariales; la dirección } \\
\text { suele ser un integrador entre la planea- } \\
\text { ción estratégica de TI y los procesos de la } \\
\text { organización integrando las TIC en todo el } \\
\text { proceso. }\end{array}$ \\
\hline
\end{tabular}

Fuente: elaboración propia a partir de Amézquita y Arango [22]

- Modelo de madurez para evaluar la capacidad de adaptación de las organizaciones al contexto del Sistema de Fabricación Inteligente (IMS): el modelo de madurez de capacidad adaptativa АСмм (Adaptive Capability Maturity Model), planteado en la investigación de El Haouzi et al. [23] en el 2013, permite evaluar la capacidad adaptativa que tienen las organizaciones a los modelos de simulación implementados en ellas, trayendo consigo dos beneficios para las empresas que lo apliquen: ayuda a avanzar hacia la construcción de un modelo de simulación que se adapte a la organización y permite seguir el progreso de los procesos de madurez en las organizaciones que lo ejecuten. A su vez, identifica una serie de elementos que contribuyen al modelado y a la simulación (gestión y procesamiento de datos, desarrollo de modelos, validación de modelos y ejecución de simulación), y a través de cinco niveles de madurez (inicial - 0 hasta optimizado - 4) pretende mejorar el proceso de adaptabilidad (Figura 2).

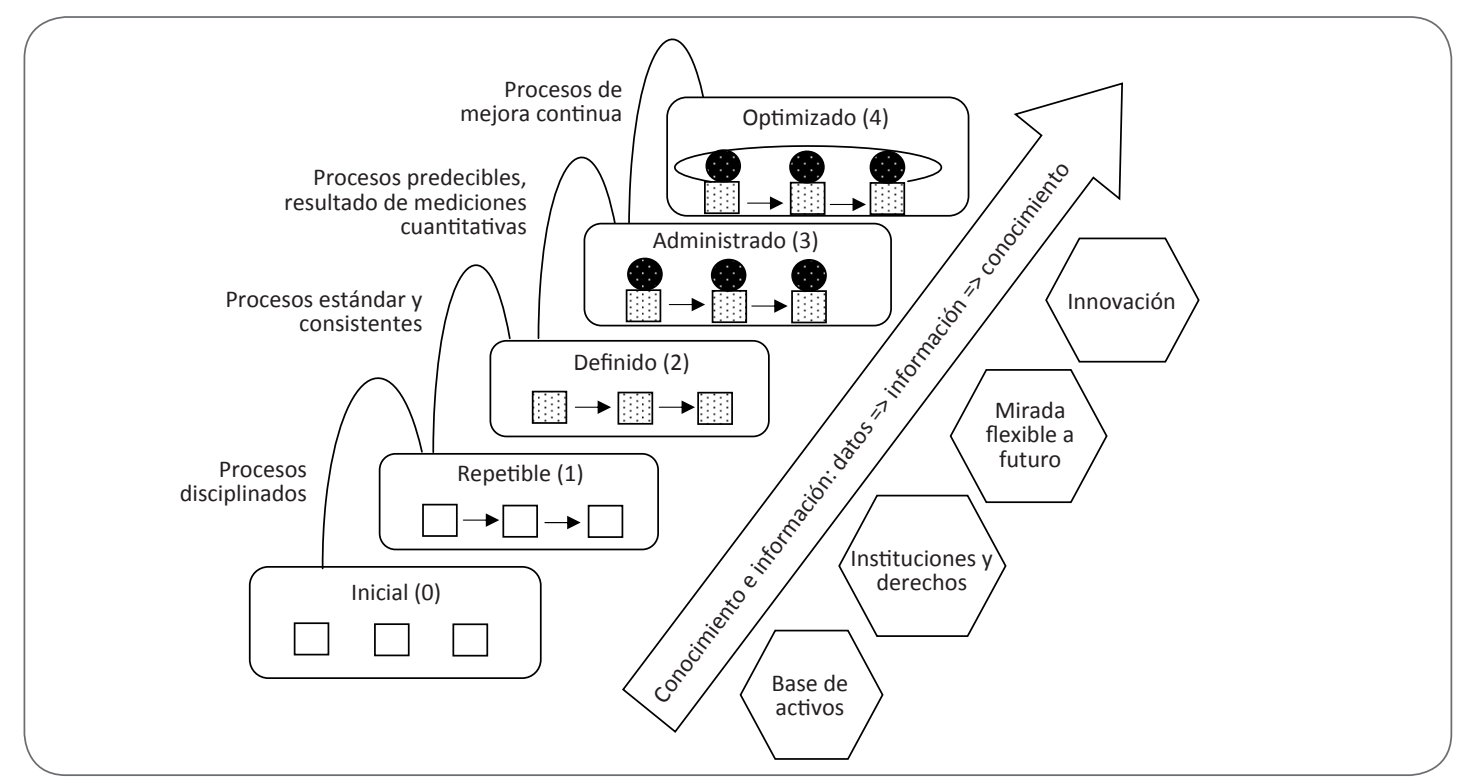

Figura 2. Modelo de madurez ACMM

Fuente: El Haouzi et al. [23] 
Cabe resaltar que los modelos de madurez anteriores son ejemplos de la aplicación de сммі en diversas áreas del conocimiento y que no existe un modelo de cMmi específico enfocado a la evaluación y mejora de los procesos de emprendimiento en las organizaciones.

\subsection{Situación del emprendimiento en las pymes}

La revisión de la literatura para esta investigación permite entender de manera general la situación del emprendimiento en las pymes colombianas a través de la contextualización del tema de emprendimiento, una mirada general de la realidad actual de las pymes y la identificación de los principales problemas para el desarrollo de procesos emprendedores en las pymes.

En primer lugar, Swanson y Devereaux [24], en su publicación del 2017 "A Theoretical Framework for Sustaining Culture: Culturally Sustainable Entrepreneurship", evidencian que el emprendimiento se ha investigado desde ciencias como la economía, la psicología y la sociología, lo cual ha generado que tenga diferentes definiciones según el punto de vista del autor. No obstante, cuando se habla sobre emprendimiento, se hace referencia a un proceso de descubrimiento, evaluación y explotación de oportunidades que está relacionado con el desarrollo de ideas innovadoras y con el crecimiento de las organizaciones.

Según las investigaciones realizadas por Saavedra-González et al. [25] en el 2017 y por Sánchez-Tovar et al. [26] en el 2015, el emprendimiento se materializa por medio de un actor llamado emprendedor, quien junto con un grupo de personas, y con la integración de recursos (materiales y monetarios principalmente), son capaces de crear nuevas organizaciones o proyectos destinados a la obtención de beneficios individuales y colectivos a través de la satisfacción de una necesidad detectada con base en la mejora continua, el desarrollo de ideas innovadoras, la generación de valor agregado (diferenciador) y el crecimiento tanto económico como estructural de la organización (Pérez [27]).

En segundo lugar, se tiene que en Colombia las pymes son definidas según la Ley 905 de 2004 [28] como: "Toda unidad de explotación económica, realizada por persona natural o jurídica, en actividades empresariales, agropecuarias, industriales, comerciales o de servicios, rural o urbana”. Según el DANE [29], en el censo económico del 2013 se estimaba que hay 1.422.117 empresas en Colombia, de las cuales el $96,4 \%$ se clasifica como mipyme (92,6\% son microempresas y 3,7 son empresas pequeñas y medianas), y que están concentradas principalmente en la ciudad de Bogotá y en departamentos como Antioquia, Valle del Cauca y Cundinamarca, según Espinosa et al. [29].

Para el caso de América Latina, la Fundación para el Desarrollo Económico Social (Fundes) señala que existen al menos dieciocho millones de empresas, de las cuales 16,6 millones son microempresas, 716,000 son empresas pequeñas y 145,000 son empresas medianas, que generan 99 millones de puestos de trabajo, como lo indican Espinosa et al. [29] en su publicación del 2015. Por ello, las pymes son reconocidas como el motor de la economía en países desarrollados y en países emergentes, debido a su gran potencial como generadoras de fuentes de empleo y crecimiento económico, según González-Amante y Mancilla-Rendón [30].

De acuerdo con lo mencionado por Martínez et al. en el 2016 [31], la Organización para la Cooperación y el Desarrollo Económicos (OCDE) señala que las microempresas y las pymes son la forma dominante de organización empresarial, pues representan hasta el 95\% de las empresas en algunos países, es decir, el 52\% del empleo y aproximadamente el 33\% del PIB entre los países miembros.

Para Minniti [32], existe un escaso número de estudios sobre las micro y pequeñas empresas en las economías emergentes. Sin embargo, referenciando lo dicho por Marulanda-Echeverry y LópezTrujillo [33] en el 2013, se sabe que estos tipos de empresas tienen claro qué competencias esenciales deben construir en el futuro para hacer frente a los nuevos productos de la implementación de estrategias a través del aprendizaje colectivo en la organización, la coordinación de habilidades de producción, la integración de múltiples corrientes tecnológicas en los productos finales y, lo más importante, un profundo compromiso a trabajar a través de los límites de la organización junto a la generación y actualización de conocimiento de manera continua. Así mismo, López-Torres et al. [34] describen que una característica de las pymes es que comúnmente obtienen un margen de utilidad mayor cuando adoptan e implementan proyectos 
emprendedores junto a la innovación como una estrategia empresarial.

Según el Global Entrepreneurship Monitor 2016 (medidor global de la actividad empresarial), en Colombia la innovación de las pymes en productos es baja, ya que más del $80 \%$ de las pymes colombianas utilizan procesos y tecnologías de hace más de cinco años, solo el $12 \%$ de los empresarios colombianos afirma que sus productos son novedosos, el $70 \%$ de las empresas pymes son del sector terciario (consumidores y outsourcing) no innovador y el $2,7 \%$ son intraempresarios o empresarios corporativos (cifra muy baja en comparación con economías basadas en innovación como Suiza, Suecia, Holanda y Estados Unidos, o Chile, México y Costa Rica para el caso de América Latina,) [35].

Como lo mencionan Herrera-Echeverri [36] y Galindo-Martín [8], existen diversos problemas de fondo que afectan el proceso emprendedor de estas pequeñas y medianas empresas, por ejemplo: los bajos niveles de educación (media, técnica y profesional), la falta de ayudas gubernamentales, el monopolio en algunos sectores productivos generado a partir de las grandes empresas y la poca preparación que un nuevo empresario tiene al momento de enfrentar los típicos problemas generados por una nueva empresa.

Por lo tanto, Alfaro-Afaro et al. [37] en su investigación del 2009 "Caracterización de la formación en emprendimiento, una alternativa para el crecimiento económico del municipio de Plato, Magdalena", plantean que se deben generar y proponer soluciones en el país que permitan el desarrollo de los procesos de emprendimiento en las empresas nacionales, así como fomentar el espíritu emprendedor en la cultura local, ya que se ha demostrado que a mayor educación emprendedora y profesional, se tomarán más decisiones acertadas respecto a cómo generar y mantener nuevas empresas, lo que trae consigo mejoras en la organización, reducción del desempleo y crecimiento económico generalizado.

\section{Metodología}

Con el fin de establecer un modelo de madurez para el emprendimiento y conforme con lo desarrollado en la presente investigación, se definió la metodología que se describe a continuación (Tabla 2).
Tabla 2. Metodología para el desarrollo del modelo

\begin{tabular}{cl}
\hline Paso & \multicolumn{1}{c}{ Descripción } \\
\hline 1 & $\begin{array}{l}\text { Identificación y definición de los principales } \\
\text { factores para procesos de emprendimiento. }\end{array}$ \\
2 & $\begin{array}{l}\text { Descripción de la propuesta de modelo de ma- } \\
\text { durez y establecimiento de niveles de madurez } \\
\text { por factores. }\end{array}$ \\
3 & $\begin{array}{l}\text { Validación del modelo de madurez mediante la } \\
\text { metodología Delphi y el juicio de expertos. }\end{array}$ \\
\hline
\end{tabular}

Fuente: elaboración propia

\subsection{Principales factores para procesos de emprendimiento}

Acs et al. [38], en el 2012, definieron que la importancia de determinar los factores que son relevantes en el proceso de emprendimiento empresarial se basa en la necesidad de medir el impacto de este tipo de actividad a nivel social y económico. Por tal motivo, en el 2006 la ocDE creó un Programa de Indicadores de Emprendimiento de la OCDE-Eurostat (Entrepreneurship Indicators Programme - EIP) presentado en el libro Entrepreneurship at a Glance, publicado desde el 2011, que compila datos internacionales y muestra mediante estadísticas la información principal sobre el espíritu empresarial y emprendedor de los países que hacen parte de esta organización.

El EIP [39] define el emprendimiento como: "El fenómeno asociado a actividad emprendedora, que es la acción humana que busca la generación de valor, a través de la creación o expansión de la actividad económica, identificando y explotando nuevos productos, procesos o mercados", y entiende la actividad emprendedora como un proceso que no se genera únicamente en las empresas nuevas, sino que también se puede presentar a lo largo del ciclo de vida de cualquier empresa.

Los indicadores básicos mostrados en Entrepreneurship at a Glance 2016 [39] evidencian que la medición del espíritu emprendedor debe ser integral. Por tanto, el EIP reconoce que no puede existir solo un indicador que permita evaluar de manera general la actividad emprendedora y proporciona una medición más detallada de este fenómeno, el cual vincula diversos factores que son propios de la actividad empresarial, incluyendo temas no solo de 
tipo económico, sino además político, social y cultural, como registra Klein [40]. Adicionalmente, la OCDE [39] en el 2016 incluyó a las pymes con el fin de obtener datos estadísticos en cuanto a la creación de empleo.

De acuerdo con los indicadores que propone el EIP frente a la medición del emprendimiento y las condiciones actuales de las pymes colombianas —según señala Varela-Villegas [4]—, esta investigación propone los siguientes factores que permiten la construcción y evaluación del "Modelo de madurez para los procesos de emprendimiento en las pymes colombianas": condiciones del mercado, aspectos económicos y financieros, creación y difusión del conocimiento, tecnologías de la información y la comunicación, y perfil del emprendedor (líder del proyecto de emprendimiento).

Cada uno de estos factores encierra un grupo de elementos que complementan y permiten la evaluación concreta del modelo, por lo cual a continuación se realizará una breve descripción de cada factor, los elementos que deben tenerse en cuenta y la importancia que tienen para el proceso de emprendimiento en las pymes (Figura 3 y Tabla 3 ).

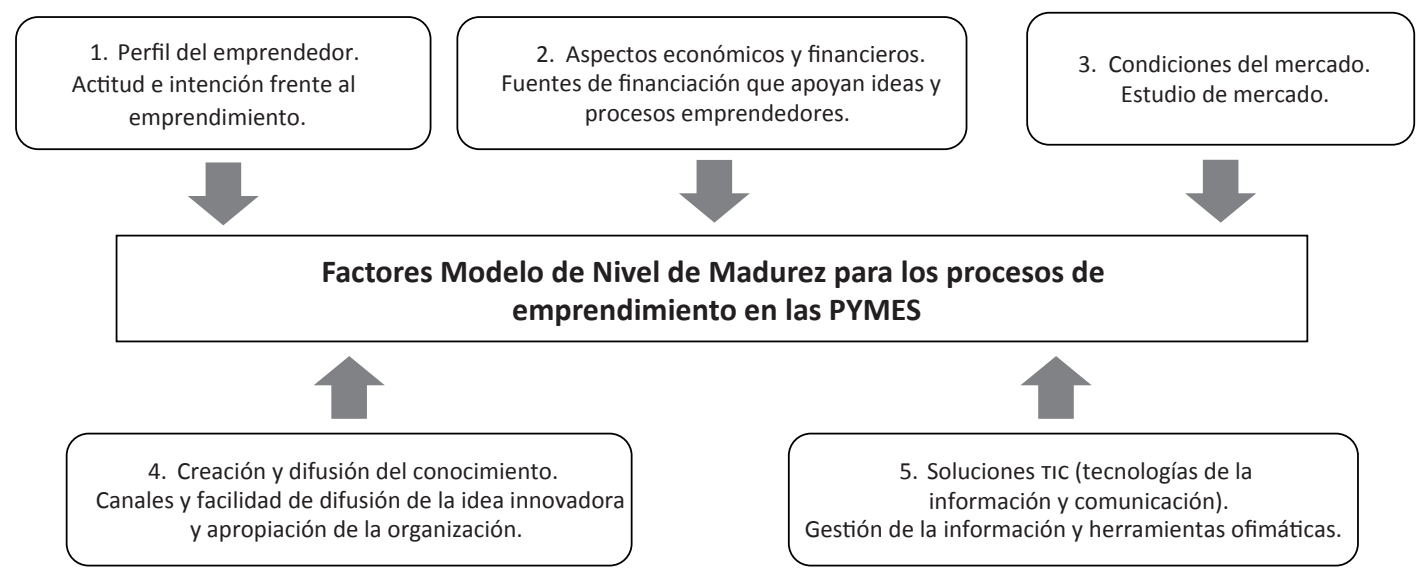

Figura 3. Factores de nivel de madurez para los procesos de emprendimiento en las pymes Fuente: elaboración propia a partir de la oCDE [39]

Tabla 3. Definición de los cinco factores del modelo de nivel de madurez

\begin{tabular}{ll}
\hline \multicolumn{1}{c}{ Factor } & \multicolumn{1}{c}{ Definición } \\
\hline 1. Perfil del emprendedor & $\begin{array}{l}\text { El emprendedor es aquel que se diferencia en el mercado por ser quien lanza un nuevo pro- } \\
\text { ducto o servicio aprovechando al máximo las oportunidades desapercibidas por medio de } \\
\text { su osadía e imaginación. Posee las siguientes características: habilidades analíticas, trabajo } \\
\text { en equipo (liderazgo) [41], responsabilidad, perseverancia, optimismo, un alto grado de } \\
\text { autoconfianza y cuenta con formación profesional y experiencia [42]. }\end{array}$ \\
& $\begin{array}{l}\text { Las empresas en general necesitan recursos financieros para crecer y sobrevivir. Las fuentes } \\
\text { de financiación pueden ser externas (como préstamos, aportaciones de capital, subsidios } \\
\text { gubernamentales o de entidades privadas) o internas (como flujos de efectivo generados o }\end{array}$ \\
y financieros & $\begin{array}{l}\text { fondos propios) [43]. La inversión en emprendimiento debe entenderse como "un negocio } \\
\text { en el que hay rendimientos crecientes" [44]. }\end{array}$ \\
& $\begin{array}{l}\text { La adquisición de información de las condiciones del mercado se ha convertido en un } \\
\text { prerrequisito para las organizaciones que quieren crear su estrategia de emprendimiento }\end{array}$ \\
3. Condiciones del & [45]. Las empresas, antes de iniciar cualquier proyecto, necesitan cuantificar el tamaño del \\
mercado & mercado, saber quiénes serán sus clientes y cuáles son necesidades, y qué características y \\
& funcionalidades puede brindar su nuevo producto y/o servicio, entre otros aspectos [46]. \\
\hline
\end{tabular}


(viene)

\begin{tabular}{ll}
\hline \multicolumn{1}{c}{ Factor } & \multicolumn{1}{c}{ Definición } \\
\hline $\begin{array}{l}\text { 4. Creación y difusión } \\
\text { del conocimiento }\end{array}$ & $\begin{array}{l}\text { La creación y difusión del conocimiento en las empresas permite: el intercambio de } \\
\text { conocimientos e ideas, espacios para la generación de conocimiento, recombinación e } \\
\text { integración de conocimientos, análisis de éxitos y estudio de fallas, el crecimiento de la } \\
\text { cultura organizacional (valores corporativos, trabajo en equipo, compromiso, disciplina y } \\
\text { calidad), y comunicación con fuentes internas y externas (proveedores, aliados) [47]. }\end{array}$ \\
& $\begin{array}{l}\text { Las Tic comprenden el conjunto de herramientas, soportes y canales que permiten acceso, } \\
\text { adquisición, producción, almacenamiento, tratamiento, comunicación, registro y presen- } \\
\text { 5. Solución de información y datos, principalmente en los campos de la informática, internet }\end{array}$ \\
& y telecomunicaciones [48]. Las Tic permiten, facilitan y alteran una amplia gama de acti- \\
& vidades (desarrollo de procesos emprendedores, tecnológicos y productivos) y estructura \\
& organizacional, con ayuda del recurso humano o los miembros de la organización [49].
\end{tabular}

Fuente: elaboración propia

\subsection{Descripción de la propuesta de modelo de madurez para los procesos de emprendimiento}

A partir de los cinco factores establecidos antes, se presenta la propuesta del "Modelo de nivel de madurez para los procesos de emprendimiento en las pymes colombianas". Este modelo permite realizar un diagnóstico que sirve como fundamento para la generación de nuevas estrategias en cuanto al emprendimiento para pymes ya constituidas.

El modelo integra los factores y define las características que debe tener cada uno de los niveles de madurez. Para la implementación del modelo en las empresas, se sugiere contactar a una persona con conocimiento en temas de emprendimiento y modelos de madurez, quien será la encargada de establecer en qué nivel de madurez se encuentra cada factor (teniendo en cuenta que para avanzar de nivel se deben cumplir con las condiciones del nivel o los niveles inferiores), y al final determinará a través del modelo en qué nivel de madurez están los procesos de emprendimiento en la organización.

Para efectos del artículo, se presenta el análisis de uno de los factores definidos en cada uno de sus niveles, dicho proceso se aplicó de manera similar para los otros cuatro factores:

Factor: Condiciones del mercado.

Nivel 1: Inicial. No se cuenta con un estudio o análisis que permita conocer las condiciones externas del mercado, lo cual dificulta el desarrollo del proceso de emprendimiento.
Nivel 2: Gestionado. Se tiene un conocimiento básico acerca de las condiciones del mercado, que permite establecer el tipo y las características del producto y/o servicio que se van a desarrollar a través del proceso de emprendimiento (ejemplo: el uso, la forma, el diseño, el empaque, la materia prima, el precio, el proceso productivo y la accesibilidad).

Nivel 3: Definido. La empresa realiza un análisis o estudio de mercado integral que proporciona un mayor conocimiento acerca de las condiciones del mercado, lo cual facilita la toma de decisiones en el proceso de emprendimiento.

Nivel 4: Administrado. Al tener un conocimiento claro sobre las condiciones del mercado, la empresa reconoce la ventaja competitiva que genera su proceso de emprendimiento frente a la competencia (ejemplo: liderazgo en costos o exclusividad).

Nivel 5: Optimizado. A través del conocimiento de las condiciones del mercado, la empresa puede establecer objetivos y alianzas estratégicas que permiten la mejora continua de sus procesos de emprendimiento (ejemplo: outsourcing y benchmarking).

Dentro del modelo, a cada factor y nivel se les asignó un peso porcentual. Estos pesos fueron asignados a partir de la investigación realizada, teniendo en cuenta las características y variables que van a ser evaluadas por cada factor. El Perfil del emprendedor es el factor con mayor relevancia (40\%), dado que para los procesos de emprendimiento en las organizaciones es necesario que haya personas con espíritu emprendedor que generen, gestionen y lleven 
a cabo nuevos proyectos e ideas. En segundo lugar, con un mismo peso porcentual se encuentran los factores de Condiciones del mercado (20\%) y Creación y difusión del conocimiento (20\%), debido a que para llevar a cabo procesos emprendedores es importante conocer a qué se está enfrentando la organización y cuál es el posible impacto que puede tener un proyecto determinado por medio de valores organizacionales propios, como aprendizaje organizacional, comunicación flexible, procesos de $\mathrm{I}+\mathrm{D}+\mathrm{i}$ e integración entre los actores de la cadena de valor. Por último, tienen un menor peso porcentual los factores de Aspectos económicos (10\%) y Soluciones TIC (10\%), que también son relevantes en la realización de procesos emprendedores, ya que permiten la mejora y continuidad de estos procesos dentro de las organizaciones siendo factores de apoyo en la gestión del emprendimiento (Tabla 4).

Tabla 4. Peso porcentual de los factores del modelo de nivel de madurez

\begin{tabular}{lc}
\hline \multicolumn{1}{c}{ Factores } & Peso porcentual \\
\hline 1. Perfil de emprendedor & $40 \%$ \\
2. Condiciones de mercado & $20 \%$ \\
3. Difusión de conocimiento & $20 \%$ \\
4. Aspectos económicos & $10 \%$ \\
5. Soluciones Tic & $10 \%$ \\
\hline
\end{tabular}

Fuente: elaboración propia

El peso de los cinco niveles definidos en el modelo de madurez propuesto se asignó de forma acumulativa: el nivel 1 es la categoría más baja, pues los procesos no se encuentran definidos dentro de la organización, y el nivel 5 es la categoría más alta, que cuenta con la mejora continua de todos los procesos (Tabla 5).

Tabla 5. Peso porcentual de los niveles del modelo de madurez

\begin{tabular}{lc}
\hline \multicolumn{1}{c}{ Nivel } & Peso porcentual \\
\hline Nivel 1: Inicial & $0 \%$ \\
Nivel 2: Gestionado & $25 \%$ \\
Nivel 3: Definido & $50 \%$ \\
Nivel 4: Administrado & $75 \%$ \\
Nivel 5: Optimizado & $100 \%$ \\
\hline
\end{tabular}

Fuente: elaboración propia
Al ser un modelo que funciona de forma acumulativa, para poder llegar al nivel 5 de madurez es necesario que las características y condiciones de los otros niveles hayan sido aprobadas.

\subsection{Validación del modelo: metodología Delphi y juicio de expertos}

La metodología Delphi y el juicio de expertos fueron los métodos utilizados para la validación del modelo propuesto por esta investigación, dado que son herramientas que facilitan el análisis y la mejora de los temas que se están evaluando por un experto. Según Mengual [50], se entiende por experto "tanto al individuo como al grupo de personas que son capaces de proporcionar valoraciones fiables sobre un problema en cuestión, y al mismo tiempo, hacen recomendaciones para el mejoramiento de este".

La metodología Delphi es una técnica de recogida de datos usada para poner de acuerdo a un grupo de expertos dispersos geográficamente sobre un tema de interés para el investigador, la cual tiene cuatro características clave: anonimato, iteración, retroalimentación controlada y agregación estadística de un grupo de respuestas, según Zartha-Sossa et al. [51]. Los siguientes ítems describen la metodología Delphi y el juicio de expertos, aplicados en esta investigación:

1. Fase preparatoria (formatos de instrucciones, cuestionarios, definición del problema y objetivos).

2. Selección de los expertos.

3. Fase de inducción (explicar el contexto del tema a evaluar).

4. Realización de la consulta a los expertos.

5. Procesamiento estadístico de los resultados de la ronda.

6. Retroalimentación de los resultados del procesamiento de las respuestas.

7. (Los pasos 3,4 y 5 se repetirían a lo largo de tantas rondas como indique el diseño del estudio).

8. Fase de análisis (se analiza lo realizado durante las rondas y el método en general).

9. Informe de resultados [52].

El grupo de expertos seleccionado para la evaluación y mejora del modelo son personas con trayectoria y conocimientos cualificados sobre emprendimiento y modelos de madurez, quienes 
pueden dar información, evidencia, juicios y valoraciones debido a su experiencia profesional y académica.

Aplicando la metodología Delphi y el juicio de expertos, se realizó un cuestionario para la validación del coeficiente de competencia experta $(K)$, en el que participaron diez personas con algún conocimiento sobre los temas de emprendimiento $\mathrm{y}$ modelos de madurez. Los resultados de esta prueba permitieron escoger cinco expertos con un (K) superior a 0,8 (alta influencia) para la validación del modelo propuesto por esta investigación (Tabla 6).

Para la validación por parte de los cinco expertos, se elaboró un cuestionario con ocho preguntas que evaluaban de manera específica: diseño, aplicabilidad, claridad, relevancia, coherencia y comprensión del modelo en general. Con el fin de facilitar el análisis estadístico de las respuestas, se estableció como estándar la escala Likert para cada pregunta con una calificación de 1 a 5 , siendo 1 totalmente de acuerdo (100\%) y 5 totalmente en desacuerdo (0\%) (Tabla 7).

\section{Resultados}

Tras aplicar los cuestionarios elaborados, las respuestas dadas por cada experto a las preguntas planteadas para la validación del modelo según la escala Likert se muestran en la tabla 8.
Tabla 7. Peso porcentual de los valores de la escala Likert

\begin{tabular}{lcc}
\hline \multicolumn{1}{c}{ Escala Likert } & Calificación & Porcentaje \\
\hline Totalmente de acuerdo & 1 & $100 \%$ \\
De acuerdo & 2 & $75 \%$ \\
$\begin{array}{l}\text { Ni de acuerdo ni en } \\
\text { desacuerdo }\end{array}$ & 3 & $50 \%$ \\
$\begin{array}{l}\text { En desacuerdo } \\
\text { Totalmente en desacuerdo }\end{array}$ & 4 & $25 \%$ \\
\hline
\end{tabular}

Fuente: elaboración propia

A partir de las valoraciones anteriores, se procedió a realizar un análisis estadístico con algunas medidas de tendencia central que reflejaron una preferencia en las respuestas por parte de los expertos hacia "totalmente de acuerdo" o "de acuerdo" con el modelo (Tabla 9).

Finalmente, con los porcentajes asignados a cada respuesta se halló el porcentaje de aceptación por cada experto, obteniendo como resultado general el 83,75\% de aceptación, lo que significa una aprobación del modelo. Sin embargo, los expertos sugirieron algunas mejoras en cuanto a: modificar los porcentajes asignados a los factores y niveles, realizar una aclaración sobre quién y cómo debe aplicarse el modelo, y plantear estrategias de mejora cuando el resultado indique que la organización se

Tabla 6. Resultado de la validación de coeficiente experto

\begin{tabular}{|c|c|c|c|c|}
\hline \multicolumn{5}{|c|}{ Evaluación de coeficiente experto } \\
\hline Experto & $\begin{array}{c}\text { Coeficiente de } \\
\text { conocimiento (KC) }\end{array}$ & $\begin{array}{c}\text { Coeficiente de } \\
\text { argumentación (KA) }\end{array}$ & $\mathrm{K}$ & Influencia del experto \\
\hline Participante 1 & 0,5 & 0,6 & 0,55 & Baja influencia \\
\hline Participante 2 & 0,7 & 1 & 0,85 & Alta influencia \\
\hline Participante 3 & 0,9 & 1 & 0,95 & Alta influencia \\
\hline Participante 4 & 0,8 & 0,9 & 0,85 & Alta influencia \\
\hline Participante 5 & 0,8 & 0,9 & 0,85 & Alta influencia \\
\hline Participante 6 & 0,5 & 0,7 & 0,6 & Baja influencia \\
\hline Participante 7 & 0,9 & 1 & 0,95 & Alta influencia \\
\hline Experto 8 & 0,5 & 0,8 & 0,65 & Baja influencia \\
\hline Experto 9 & 0,6 & 0,8 & 0,7 & Media influencia \\
\hline Experto 10 & 0,6 & 0,8 & 0,7 & Media influencia \\
\hline
\end{tabular}


encuentra entre los niveles de madurez 1, 2, 3 y 4 (Figura 4).

Después de obtener la aceptación del modelo, se realizaron los cambios sugeridos, mejorando la aplicabilidad y funcionalidad del modelo para que pueda cumplir con el objetivo de esta investigación.

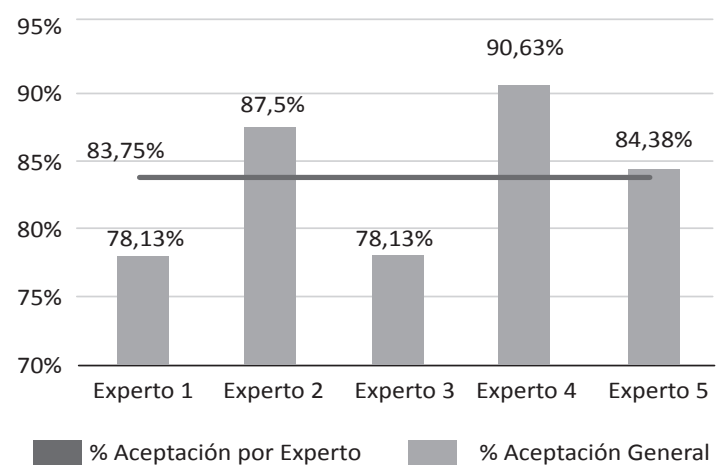

Figura 4. Porcentaje de aceptación de cada experto frente al promedio general

Fuente: elaboración propia

\section{Discusión}

Como se mostró en esta investigación, los modelos CMM tienen muchas aplicaciones en diversas áreas del conocimiento, lo cual permite que se puedan evaluar procesos en organizaciones con enfoques económicos o sociales múltiples, evidenciando que la metodología y estructura del modelo tiene gran aplicabilidad y uso. Sin embargo, estos modelos han sido desarrollados en gran medida para la ingeniería de software y aún la metodología no ha sido adaptada y/o utilizada en todos los campos que podría abarcar; esto permite abrir el panorama para la construcción de diferentes modelos de madurez enfocados a la mejora de los procesos organizacionales. Con base en lo anterior, se espera que se puedan desarrollar futuras investigaciones sobre el diseño y la aplicabilidad de los modelos CMMI en otros ámbitos.

La investigación realizada también permitió conocer los aspectos principales que se deben considerar para construir o desarrollar un modelo de madurez que cumpla con las características básicas que han usado sus creadores y otros autores. Es

Tabla 8. Ponderación de preguntas por cada experto según respuesta Likert

\begin{tabular}{l|cccccccc}
\hline Expertos & Preguntas & P2 & P3 & P4 & P5 & P6 & P7 & P8 \\
\hline Experto 1 & 2 & 2 & 2 & 1 & 1 & 4 & 1 & 2 \\
Experto 2 & 2 & 1 & 2 & 1 & 1 & 1 & 2 & 2 \\
Experto 3 & 2 & 2 & 2 & 1 & 2 & 2 & 2 & 2 \\
Experto 4 & 1 & 2 & 2 & 1 & 1 & 1 & 1 & 2 \\
Experto 5 & 1 & 2 & 2 & 1 & 1 & 2 & 2 & 2 \\
\hline
\end{tabular}

Fuente: elaboración propia

Tabla 9. Medidas de tendencia central por cada pregunta para todos los expertos

\begin{tabular}{l|cccccccc}
\hline Medida & P1 & P2 & P3 & P4 & P5 & P6 & P7 & P8 \\
\hline Moda & 2 & 2 & 2 & 1 & 1 & 1 & 2 & 2 \\
Media & 1,6 & 1,8 & 2 & 1 & 1,2 & 2 & 1,6 & 2 \\
Varianza & 0,3 & 0,2 & 0 & 0 & 0,2 & 1,5 & 0,3 & 0 \\
Desviación estándar & 0,54 & 0,44 & 0 & 0 & 0,447 & 1,225 & 0,548 & 0 \\
\hline
\end{tabular}

Fuente: elaboración propia 
decir que cuando se quiera utilizar la metodología CMMI para la elaboración de modelos de madurez, es importante seguir el esquema del modelo, que puede enfocarse en distintos temas, pero que no puede perder su esencia que establece niveles y criterios de evaluación para cada nivel. El modelo de nivel de madurez para los procesos de emprendimiento en las pymes colombianas se construyó con base en los modelos consultados a fin de no modificar la estructura planteada por estos, logrando obtener las principales características de los CMMI con el enfoque hacia la evaluación de los procesos de emprendimiento.

Es necesario reiterar la importancia que tienen los procesos de emprendimiento en las empresas y la necesidad que existe de evaluarlos continuamente, con el fin de que sea un proceso específico, ordenado y que genere los resultados esperados. Para asumir procesos de emprendimiento organizacionales como estrategia para la mejora continua, la organización debe contar principalmente con un grupo de emprendedores que sean capaces de gestionar, administrar y controlar dichos procesos, puesto que el emprendimiento no es simplemente generar ideas o proyectos nuevos, sino que es todo un proceso que tiene fases, objetivos y actividades que deben ser ejecutadas en un tiempo determinado.

Así mismo, en el estudio del emprendimiento se han generado diferentes avances e investigaciones que permiten ver la importancia que tienen este tipo de procesos en el crecimiento y fortalecimiento económico de las empresas. Para las pymes colombianas, integrar procesos de emprendimiento en sus organizaciones es un desafío actual que requiere de grandes cambios en su estructura organizacional y la inversión de recursos en I+D+i que estén enfocados en la mejora continua de sus procesos y en el desarrollo de nuevos productos.

El modelo planteado en esta investigación se ha construido de manera conceptual y se espera que sea una aproximación apropiada a la construcción de un modelo сммі que permita evaluar diferentes procesos organizacionales y mejore su nivel de madurez. Este modelo está sujeto a estudios, pruebas y mejoras adicionales.

\section{Conclusiones}

El modelo CMMI planteado en la investigación "Modelo de nivel de madurez para los procesos de emprendimiento en las pymes colombianas" incluye los factores principales que deben ser tomados en cuenta para que exista un proceso emprendedor en la organización. Estos factores se adaptan a las necesidades actuales de las pymes, ya que evalúan aspectos como: la inversión financiera, el conocimiento del mercado, la capacidad de creación y difusión del conocimiento, las herramientas tecnológicas y el capital humano para promover la actividad emprendedora en la organización. Este modelo fue validado a través de la metodología Delphi y el método de juicio de expertos, logrando un porcentaje de aceptación de 83,75\% que demuestra que contiene los elementos básicos para evaluar los procesos de emprendimiento. No obstante, para su total aprobación requiere sobre todo que sea probado en pymes con el fin de verificar su completa aplicabilidad y proponer mejoras específicas para el modelo.

El modelo de madurez propuesto permite realizar un diagnóstico sobre el estado de los procesos de emprendimiento dentro de la organización. Al aplicar el modelo, se puede determinar en qué nivel de madurez está cada factor, con el fin de proponer acciones de mejora que fortalezcan su proceso de emprendimiento. Frente a este objetivo y evaluando el factor descrito en este documento (Condiciones del mercado), se proponen las siguientes acciones de mejora para avanzar al nivel superior:

Nivel 1: realizar un estudio o análisis para conocer condiciones externas del mercado, como: tipo de cliente, competencia, estructura del mercado, facilidad de ingreso y permanencia en el mercado, y bienes sustitutos y complementarios, entre otras.

Nivel 2: aplicar el conocimiento acerca de las condiciones del mercado generando estrategias que incluyan el desarrollo de productos o servicios innovadores que tengan un alcance definido, características diferenciadoras y faciliten los planes de mercadeo.

Nivel 3: con los resultados y análisis del estudio de mercado, la organización debe generar la ventaja competitiva que diferencie sus productos o servicios en cuanto a liderazgo en costos, exclusividad y preferencia de los clientes.

Nivel 4: encontrar diferentes opciones para mejorar el posicionamiento estratégico de la empresa en el mercado, a través de nuevas alianzas con proveedores y competidores que faciliten 
el desarrollo de ideas innovadoras e impulsen el emprendimiento entre las organizaciones.

Esta investigación es una primera aproximación a los CMMI para temas de emprendimiento, y requiere de mayor profundización y futuras investigaciones que realicen un análisis sobre aplicaciones reales de estos modelos de madurez en las pequeñas y medianas empresas.

\section{Referencias}

[1] M. F. Ángel y D. U. Pulido, "El éxito de las pymes en Colombia: un estudio de casos en el sector salud". Estud. Gerenciales, vol. 26, no. 114, pp. 77-96, 2010, doi: https://doi.org/10.1016/S0123-5923(10) 70103-0.

[2] L. Salas-Arbeláez, M. García Solarte y G. Murillo Vargas, "Efecto de la cultura organizacional en el rendimiento de las pymes de Cali". Suma Negocios, vol. 8 , no. 18 , pp. $88-95,2017$, doi: https://doi.org/10.1016/j.sumneg.2017.11.006

[3] J.-P. Lis-Gutiérrez, O. Palencia-Fajardo y M. Gaitán-Angulo, "Caracterización de las pymes de confecciones en Neiva (Colombia)". Suma Negocios, vol. 8 , no. 17 , pp. 57-62, 2017, doi: https://doi.org/10.1016/j.sumneg.2017.03.002

[4] R. O. Varela-Villegas, "Colombia Small- and Medium-Sized Enterprise's 70 Years of Progress: What's Next?". Small Enterp. Res., vol. 23, no. 3, pp. 302-315, 2016, doi: https://doi.org/10.1080/13215906.2016.1 269241

[5] D. García-Pérez de Lema, E. J. Gálvez-Albarracín y G. Maldonado-Guzmán, "Efecto de la innovación en el crecimiento y el desempeño de las Mipymes de la Alianza del Pacífico. Un estudio empírico". Estud. Gerenciales, vol. 32, no. 141, pp. 326-335, 2016, doi: https://doi.org/10.1016/j.estger.2016.07.003

[6] D. M. Escandón Barbosa y A. Hurtado Ayala, "Los determinantes de la orientación exportadora y los resultados en las pymes exportadoras en Colombia". Estud. Gerenciales, vol. 30, no. 133, pp. 430-440, 2014, doi: https://doi.org/10.1016/j.estger.2014.05.002

[7] F. Pereira, Reporte GEM. Cali 2010-2011. Cali: Universidad Javeriana, 2011 [En línea]. Disponible en: https://www.icesi.edu.co/cdee/images/informes/ regionales/Cali-2010-2011.pdf

[8] M. Á. Galindo-Martín, M. T. Méndez-Picazo y M. S. Castaño-Martínez, "Crecimiento, progreso económico y emprendimiento". J. Innov. Knowl., vol. 1, no. 1, pp. 62-68, 2016, doi: https://doi.org/10.1016/j. jik.2016.01.006
[9] H. Kantis, P. Angelelli y V. Moori Koenig, Desarrollo emprendedor. América Latina y la experiencia internacional. Washington D.C.: Banco Interamericano de Desarrollo, 2004 [En línea]. Disponible en: http:// scholar.google.com/scholar?hl=en\&btnG=Search\&q=intitle:Desarrollo+emprendedor\#0

[10] Software Engineering Institute (sEI), Capability Maturity Model $^{\circledast}$ Integration (CMMI), Version 1.1. Pittsburgh: Carnegie Mellon University, 2002.

[11] F. Backlund, D. Chronéer y E. Sundqvist, "Project Management Maturity Models - A Critical Review: A Case Study within Swedish Engineering and Construction Organizations". Procedia Soc. Behav. Sci., vol. 119, no. 19, pp. 837-846, 2014, doi: https:// doi.org/10.1016/j.sbspro.2014.03.094

[12] O. R. Puello, "Model Verification and Validation Based on сммі". Rev. Investig. innovación e Ing., vol. 1, no. 1, pp. 20-27, enero-junio 2013, doi: 10.17081/ invinno.1.1.2068

[13] R. Kishore, M. E. Swinarski, E. Jackson y H. R. Rao, "A Quality-Distinction Model of Iт Capabilities: Conceptualization and Two-Stage Empirical Validation Using CMmi Processes". IEeE Trans. Eng. Manag., vol. 59, no. 3, pp. 457-469, 2012, doi: 10.1109/ TEM.2011.2165287

[14] J. Sutherland, C. R. Jakobsen y K. Johnson, "Scrum and смMI Level 5: The Magic Potion for Code Warriors", en Proceedings of the 41st Annual Hawaii International Conference on System Sciences (HICSS 2008). Waikoloa: IEEE, 2008, doi: 10.1109/ HICSS.2008.384

[15] A. M. L. De Vasconcelos, J. L. De La Vara, J. Sánchez y Ó. Pastor, "Towards сммI-Compliant Business Process-Driven Requirements Engineering", en 2012 Eighth International Conference on the Quality of Information and Communications Technology. Lisboa: IEEE, 2012, doi: 10.1109/QUATIC.2012.61

[16] J. E. Peña-Forero y H. S. Aguirre-Mayorga, "Propuesta de diseño de un modelo de gestión basado en la integración de herramientas de tecnología de información (Iт) a partir del análisis del estado de madurez de procesos (СммI) para micro, pequeñas y medianas empresas (mipymes) en Bogotá, caso de estudio". J. Chem. Inf. Model., vol. 53, no. 9, pp. 1689-1699, 2013 [En línea]. Disponible en: http:// hdl.handle.net/10554/15522

[17] J. Mejía, M. González y M. Muñoz, "Organization of the Process Areas of CMMI-Dev v1.3 Level 2 Through of its Dependencies", en 2017 12th Iberian Conference on Information Systems and Technologies (CISTI), Lisboa: IEEE, 2017, doi: 10.23919/CISTI.2017.7975919

[18] H. Arboleda, A. Paz y R. Casallas, "Metodología para implantar el Modelo Integrado de Capacidad 
de Madurez en grupos pequeños y emergentes". Estud. Gerenciales, vol. 29, no. 127, pp. 177-188, 2013, doi: https://doi.org/10.1016/j.estger.2013.05.006

[19] C. M. Zapata Jaramillo, J. Valderrama Betancur y L. D. Jiménez Pinzón, "Representation of CMMI-DEV Practices in the Semat Kernel". IEEE Lat. Am. Trans., vol. 13 , no. 10 , pp. $3476-3481$, 2015, doi: 10.1109/ TLA.2015.7387257

[20] J. Garzás, C. M. Fernández y M. Piattini, “Una aplicación de la norma Iso/IEC 15504 para la evaluación por niveles de madurez de Pymes y pequeños equipos de desarrollo". REICIS Rev. Española Innovación, Calid. e Ing. del Softw., vol. 5, no. 2, 2009 [En línea]. Disponible en: http://www.redalyc.org/articulo.oa? id $=92217153012$.

[21] M. M. L. Petrie, V. H. M. García y G. M. Giraldo, "Modelo de Registro y Acreditación de Instituciones de Educación Superior basado en el modelo CMMI", en VII LACCEI Lat. Am. Caribb. Conf. Eng. Technol., San Cristóbal, Venezuela, 2009 [En línea]. Disponible en: laccei.org/LACCEI2009-Venezuela/Papers/ Acc116_LarrondoPetrie.pdf

[22] J. Amezquita y P. Arango, Aplicación del modelo de madurez de alineación estratégica de Luftman en el sector bancario. Bogotá: Universidad del Rosario, 2014 [En línea]. Disponible en: http://repository. urosario.edu.co/bitstream/handle/10336/8684/ 1026269722-2014.pdf? sequence $=1$.

[23] H. B. El Haouzi, A. Thomas y P. Charpentier, "Toward Adaptive Modelling \& Simulation for IMs: The Adaptive Capability Maturity Model and Future Challenges". IFAC Proceedings Volumes, vol. 46, no. 7, pp. 174-179, mayo 2013, doi: https://doi. org/10.3182/20130522-3-BR-4036.00104

[24] K. K. Swanson y C. Devereaux, "A Theoretical Framework for Sustaining Culture: Culturally Sustainable Entrepreneurship", Annals of Tourism Research, vol. 36, pp. 78-88, enero 2017, doi: https://doi.org/10.1016/j.annals.2016.12.003

[25] R. Saavedra-González, B. León-Valle, M. González-Santos, W. Nuñez de la Cruz, C. Félix-Silva y B. Reyes-Tomalá, "Emprendimiento femenino en el desarrollo comunitario del barrio Los Matías Cantón, Salinas, provincia Santa Elena”. Revista CTU Científica y Tecnológica UPSE, vol. 4, no. 1, abril 2017, doi: https://doi.org/10.26423/rctu.v4i1.242

[26] Y. Sánchez-Tovar, F. García Fernández y J. E. Mendoza-Flores, "La capacidad de innovación y su relación con el emprendimiento en las regiones de México". Estud. Gerenciales, vol. 31, no. 136, pp. 243-252, 2015, doi: https://doi.org/10.1016/j.estger.2015.04.001

[27] I. Pérez, "Entrepreneurship as a Euphemism for Self-Employment”. Procedia - Soc. Behav. Sci., vol.
139, pp. 128-136, 2014, doi: https://doi.org/10.1016/ j.sbspro.2014.08.042

[28] Colombia, Congreso de la República (2 ago. 2004). Ley 905, Por medio de la cual se modifica la Ley 590 de 2000 sobre promoción del desarrollo de la micro, pequeña y mediana empresa colombiana y se dictan otras disposiciones [En línea]. Disponible en: http:// www.secretariasenado.gov.co/senado/basedoc/ ley_0905_2004.html

[29] F. Romero Espinosa, Z. A. Melgarejo Molina y M. A. Vera-Colina, "Fracaso empresarial de las pequeñas y medianas empresas (pymes) en Colombia”. Suma Negocios, vol. 6, no. 13, pp. 29-41, 2015, doi: https:// doi.org/10.1016/j.sumneg.2015.08.003

[30] O. D. González-Amante, M. E. Mancilla-Rendón, "Propuesta de factores organizacionales en un modelo de innovación para PyMEs: empresas dedicadas a la elaboración de concreto premezclado en México", en Memorias del XVIII Concurso Lasallista de Investigación, desarrollo e Innovación CLIDi 2016. Bogotá: Universidad de La Salle, 2016, pp. 17-21 [En línea]. Disponible en: http://ojs.dpi.ulsa.mx/ index.php/Memorias_del_Concurso/article/viewFile/1021/1283

[31] A. G. Martínez, M. Rosa y L. Mejía, "Gender differences in the approval of credit to Mexican sMEs". Contaduría y Adm., vol. 61, no. 4, pp. 777-793, 2016, doi: https://doi.org/10.1016/j.cya.2015.10.002

[32] M. Minniti, "El emprendimiento y el crecimiento económico de las naciones”. Econ. Ind., no. 383, pp. 23-30, 2012 [En línea]. Disponible en: http://www. minetad.gob.es/Publicaciones/Publicacionesperiodicas/EconomiaIndustrial/RevistaEconomiaIndustrial/383/Mar\%C3\%ADa\%20Minnit.pdf_

[33] C. E. Marulanda-Echeverry y M. López-Trujillo, "La gestión del conocimiento en las PyMes de Colombia”. Knowl. Manag. Colomb. sMes., no. 38, pp. 158-170, 2013, doi: https://doi.org/10.17230/ad-minister.29.8

[34] G. C. López-Torres, G. Maldonado, S. Y. Pinzón y R. García, "Collaboration and Innovation Activities in sMEs". Contaduría y Adm., vol. 61, no. 3, pp. 568-581, 2016, doi: https://doi.org/10.1016/j.cya. 2015.05.016

[35] Global Entrepreneurship Monitor, Actividad Empresarial Colombiana. Reporte GEM Colombia 2016/2017. Cali: Universidad Javeriana, 2017 [En línea]. Disponible en: https://www.researchgate.net/ publication/317929591_Actividad_Empresarial_ Colombiana_Reporte_GEM_Colombia_20162017.

[36] H. Herrera-Echeverri, “Investigación sobre redes sociales y emprendimiento: revisión de la literatura y agenda futura”. Rev. Innovar, vol. 19, no. 33, pp. 19- 
33, enero-abril 2009 [En línea]. Disponible en: www. scielo.org.co/pdf/inno/v19n33/v19n33a02.pdf

[37] C. M. Alfaro-Alfaro, M. E. Cruzate-González, F. M. Santana-Parra, D. D. Peña-González. "Caracterización de la formación en emprendimiento, una alternativa para el crecimiento económico del municipio de Plato, Magdalena”. Revista Escenarios, vol. 14, no. 2, doi: http://dx.doi.org/10.15665/esc.v14i2.934

[38] Z. J. Acs, D. B. Audretsch, P. Braunerhjelm y B. Carlsson, "Growth and entrepreneurship". Small Bus. Econ., vol. 39, no. 2, pp. 289-300, 2012, doi: https:// doi.org/10.4337/9781781009949.00009

[39] Organización para la Cooperación y el Desarrollo Económicos (OCDE), Entrepreneurship at a Glance 2016. París: OCDE, 2016 [En línea]. Disponible en: http://www.oecd-ilibrary.org/docserver/download/ 3016021 e.pdf? expires $=1513042163 \& i d=i d \& a c c-$ name $=$ guest $\&$ checksum $=265873$ C5106D1CCA95DB94D3E3E8C0B8

[40] P. G. Klein, "Why Entrepreneurs Need Firms, and the Theory of the Firm Needs Entrepreneurship Theory". Rev. Adm. (São Paulo)., vol. 51, no. 3, pp. 323-326, 2016, doi: https://doi.org/10.1016/j. rausp.2016.06.007

[41] E. Da Silva-Añaña, F. A. Dos Anjos, M. de Lima-Pereira, "Imagem de destinos turísticos: avaliação à luz da teoria da experiência na economia baseada em serviços". Revista Brasileira de Pesquisa em Turismo, vol. 10, no. 2, pp. 107-127, 1992, doi: https://doi. org/10.7784/rbtur.v10i2.1093

[42] A. M. Ciruela-Lorenzo, M. Cuadrado-Serrán, J. J. Plaza-Angulo, "La economía social como alternativa de autoempleo. El perfil del emprendedor/a en las sociedades laborales andaluzas". Revesco Rev. Estud. Coop., vol. 122, pp. 59-85, 2016, doi: https://doi. org/10.5209/rev_reve.2016.v122.52019

[43] S. Bhattacharya y B. R. Londhe, "Micro Entrepreneurship: Sources of Finance \& Related Constraints". Procedia Econ. Financ., vol. 11, no. 14, pp. 775-783, 2014, doi: https://doi.org/10.1016/S22125671(14)00241-X

[44] J. Lerner, "The Future of Public Efforts to Boost Entrepreneurship and Venture Capital”. Small Bus. Econ., vol. 35, no. 3, pp. 255-264, 2010, doi: https:// doi.org/10.1007/s11187-010-9298-Z
[45] M. Garri y N. Konstantopoulos, "Market Information Acquisition: A Prerequisite for Successful Strategic Entrepreneurship". Procedia - Soc. Behav. Sci., vol. 73, pp. 643-651, 2013, doi: https://doi.org/10.1016/j.sbspro.2013.02.101

[46] C. E. Davis, J. J. Sluss y R. E. Bolen, "Developing an engineering and entrepreneurship collaborative project", en 2014 IEEE Frontiers in Education Conference (FIE) Proceedings. Madrid: IEEE, 2014, doi: 10.1109/FIE.2014.7044482

[47] S. A. Zahra, "Corporate Entrepreneurship as Knowledge Creation and Conversion: The Role of Entrepreneurial Hubs". Small Bus. Econ., vol. 44, no. 4, pp. 727-735, 2015, doi: https://doi.org/10.1007/s11187015-9650-4

[48] S. de Pablo-Martín, "El uso de las Tic en la gestión empresarial”, Trabajo de grado, Univ. Valladolid, Valladolid, España, 2016 [En línea]. Disponible en: http://uvadoc.uva.es/bitstream/10324/23407/1/ TFG-O 967.pdf

[49] I. Giotopoulos, A. Kontolaimou, E. Korra y A. Tsakanikas, "What Drives ICT Adoption by smes? Evidence from a Large-Scale Survey in Greece". J. Bus. Res., vol. 81, pp. 60-69, diciembre 2017, doi: https://doi.org/10.1016/j.jbusres.2017.08.007

[50] J. Cabero-Almenara y J. Barroso Osuna, "La utilización del juicio de experto para la evaluación de TIC: el coeficiente de competencia experta". Bordón, vol. 65, no. 2, pp. 75-96, 2013, doi: https://doi. org/10.13042/brp.2013.65202

[51] J. W. Zartha-Sossa, J. M. Montes-Hincapié, I. D. Toro-Jaramillo, H. S. Villada, "Método Delphi. Propuesta para el cálculo del número de expertos en un estudio Delphi sobre empaques biodegradables al 2032". Rev. Espac., vol. 35, no. 13, p. 10, 2014 [En línea]. Disponible en: https://www.re searchgate.net/file.PostFileLoader.html?id=5620 ffb35e9d9789978b4586\&assetKey=AS\%3A28517 8191728644\%401445003187777

[52] F. Hernández-García, J. I. Robaina-Castillo, "Guía para la utilización de la metodología Delphi en las etapas de comprobación de productos terminados tipo software educativo". Revista 16 de Abril, vol. 56, no. 263, pp. 26-31, 2017 [En línea]. Disponible en: http://www.rev16deabril.sld.cu/index.php/16_04/ article/view/429/pdf_125 